\title{
Design of Motion Platform for Intelligent Vehicle
}

\author{
Li Shaowei ${ }^{1}$, Cheng Hui ${ }^{1}$, Wang Shengzheng ${ }^{2+}$ \\ ${ }^{1}$ School of Mathematics and Computer Science, Jianghan University, Wuhan Hubei 430056, China \\ ${ }^{2}$ Merchant Marine College, Shanghai Maritime University, Shanghai 201306, China
}

\begin{abstract}
In order to assist the developers to set and revise PID control parameters for intelligent vehicle, a kind of simulation platform for smart car was designed, the simulation platform consists of three modules. The track module has the shape and parameters of the racetrack in the current simulation environment; The vehicle module included a motion model and parameters of the intelligent vehicle; The simulation module was used to simulate the physical operation environment and establish the coordinate information. During the operation, the running simulation module receives the vehicle movement and PID control parameters from the user, and obtains the calculation of the vehicle position and the error information with the assistance of the vehicle module. Experiments show that compared with the existing algorithms, the platform is not limited by the sensor type, which can simulate the movement state of the vehicle more realistically, and provide the practical basis for the PID parameter adjustment of the developer.
\end{abstract}

Keywords: intelligent vehicle, PID, simulation platform, modularization, motion model.

\section{Introduction}

In order to solve the shortcomings in the existing simulation platform [1-5], Tsinghua University and Shanghai Jiaotong University, respectively, based on the design of the optical camera intelligent car simulation program Plastid and Cyber-Smart [6], [7]. Shanghai Jiaotong University Lu Zhengchen et al. Used the above simulation system to design a set of intelligent vehicle simulation platform based on electromagnetic guidance [8]. In this platform, an electromagnetic sensor model and a vehicle motion model are established to help the intelligent vehicle simulation program identify the position and error information of the vehicle in the runway. But the design of the whole system does not involve the vehicle control algorithm, which means that once the vehicle from the track, the simulation program can not be based on the actual operation of the situation, correct vehicle location information; Hangzhou University of Electronic Science and Technology Wu Qiuxuan, who will Microsoft MRDS as a development platform, the design of a smart car simulation platform [9]. The core technology of this simulation platform is too dependent on the underlying development platform, and the practicality and accuracy of the whole system is not verified; foreign intelligent vehicle simulation platform is more inclined to the overall study of vehicle traffic flow, such as Yue Yu and Abdelkader El Kamel et al. Proposed a vehicle simulation model based on multi-agent [10]. In this method, the vehicle, the road and the environment are represented by the object-oriented Agent method, and the obtained Agent object is introduced into the virtual reality simulation environment. This method will focus more attention on the control of vehicle traffic flow, weakening the steering performance of the vehicle itself, is an ideal vehicle simulation model; Chungen Hung and Abdulrahman Yarali et al. Proposed a wireless positioning Of the intelligent vehicle control platform [11]. The platform uses a wireless location to inform the vehicle of the current location and guide the smart car into the next positioning point. JM Collado and Chunzhao Guo and Seiichi Mita have proposed a smart vehicle control method based on machine vision. The JM Collado and Chunzhao Guo and Seiichi Mita have proposed a smart vehicle control

\footnotetext{
+ Corresponding author. Tel.: 86-18171385977; fax: 86-27-84226927.

E-mail address:55440881@qq.com.
} 
method based on machine vision. The method has the advantages of simple and safe control algorithm, The former using the traditional lane recognition algorithm and the latter is proposed a target classification method [12], [13]. These two methods use the sensor to return information to determine the location of the lane, and real-time correction of vehicle location information.

In this paper, the simulation program is designed with sub-module object-oriented design method. It is divided into three modules: motion simulation module, vehicle module and runway module. In each of the different modules, a general-purpose four-wheel vehicle motion model, a segmented PID control algorithm, a runway parameter information and a motion simulation algorithm are established respectively. The simulation platform realizes the simulation of vehicle movement by reading the vehicle model data, runway information and PID control parameters which can be input by the user, and the motion error is reflected to the developer to assist the developers in adjusting the control parameters and the model.

\section{Design Method}

\subsection{Motion model}

In the structure, this article involves the intelligent car for the four-wheel structure, left and right rear wheels are installed with drive motor, the front wheel is connected to the steering gear is responsible for control the direction, as shown in Figure 1 for the wheelbase L, wheel spacing W, The front wheel steering angle is $\alpha$, the intelligent car movement diagram ${ }^{[14][15]}$.

According to the basic model of vehicle movement, we can see that the intelligent vehicle will be circular motion around the same circle in the state shown in Fig. 1, and the direction of the front wheel of the vehicle is the direction of its circular motion tangent. So that the tangent of the vertical line and with the rear axle extension line at a little $\mathrm{O}$, this point is the circular motion of the vehicle when the center.

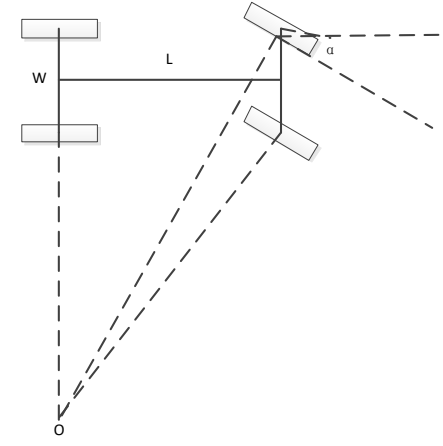

Fig. 1: Motion model for smart car.

\subsection{Differential between wheels}

The two rear wheels of the intelligent vehicle are driven by different motors, so they must adjust the speed difference according to the steering angle of the front wheel. Assuming that the rotational speed of the left and right rear wheels is $\mathrm{vl}$ and $\mathrm{vr}$ when the vehicle is turned, the right turning radius is $\mathrm{Rr}$ and the angular velocity of movement is $\omega$. According to Figure 1,

$$
\omega=\frac{v_{l}}{R_{r}+W}=\frac{v_{r}}{R_{r}}
$$

According to the basic rules of plane geometry we can see

$$
R_{r}=\frac{L}{\tan \alpha}-W
$$

General formula 1, 2 can be given the following formula 3

$$
\frac{v_{l}}{v_{r}}=1+\frac{W^{*} \tan \alpha}{L-W^{*} \tan \alpha}
$$

\subsection{Turning radius}


Smart car in the steering of the best state is to maintain the front axle center in the runway centerline, according to Figure 1 shows that the front axle center point of the turning radius and the front wheel steering angle between the following relationship

$$
R_{m}=\sqrt{L^{2}+\left(R_{r}+W / 2\right)^{2}}
$$

\subsection{Wheel grip}

Smart car in the steering due to the impact of centrifugal force, between the wheel and the ground will produce transverse friction, once the centrifugal force exceeds the maximum lateral friction, the vehicle will slip, the maximum speed of the smart car steering and turning radius and the maximum transverse friction of the wheel have the following relationship

$$
v=\sqrt{\frac{F R_{m}}{m}}
$$

Where $\mathrm{m}$ is the quality of the smart car, a fixed value, $\mathrm{Rm}$ can be obtained by the formula.

\subsection{Control model}

Intelligent vehicle control model using segmented PID control method, that is, according to the smart car in the racetrack in different locations and the current speed, select the different PID control parameters to achieve intelligent vehicle position error correction. Figure 2 shows the structure of the intelligent vehicle control model based on the classical PID control method.

PID control parameters are $\mathrm{Kp}, \mathrm{Kd}, \mathrm{Ki}$, respectively, using the first-order differential method to achieve differential numerical calculation, then you can get as shown in Figure 6 of the intelligent vehicle control model calculation equations.

$$
\begin{aligned}
& K_{p}=a * e(t) \\
& K_{i}=b * \frac{e(t)-e(t-\Delta t)}{\Delta t} \\
& K_{d}=c * \sum_{t=T_{1}}^{T_{2}} e(t) * \Delta t \\
& F=K_{p}+K_{i}+K_{d}
\end{aligned}
$$

Where $\mathrm{F}$ represents the amount of control used to adjust the servo and motor parameters.

\section{Models for Simulation Platform}

The intelligent vehicle simulation platform is realized by modular design. It is composed of three parts: running simulation module, vehicle module and track module. The logical relationship is shown in figure 2 .

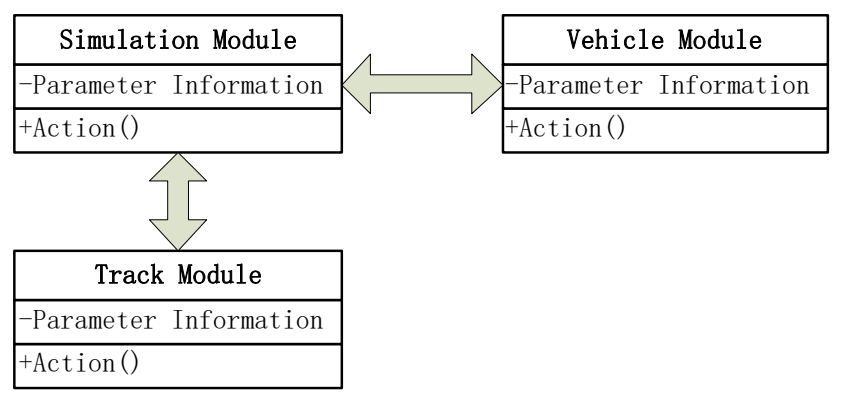

Fig. 2: Simulation modules.

\section{Simulation and Algorithm Comparison}

\subsection{Racetrack parameters}

In order to facilitate the establishment of the experimental platform, simplify the simulation calculation work, we select the racetrack parameters shown in figure 3 as the simulation experiment platform. According to Fig. 4, the track shape is a square having a corner of 90 degrees, with a straight length of $5 \mathrm{~m}$, a racetrack 
width of $40 \mathrm{~cm}$, an inner diameter of the corner fan shape of $40 \mathrm{~cm}$, and an outer diameter of $80 \mathrm{~cm}$. The left and outer sides of the racetrack coincide with the $\mathrm{Y}$ axis and the $\mathrm{X}$ axis of the Cartesian coordinate system, respectively, to facilitate the calculation of the position error ${ }^{[16-18]}$.

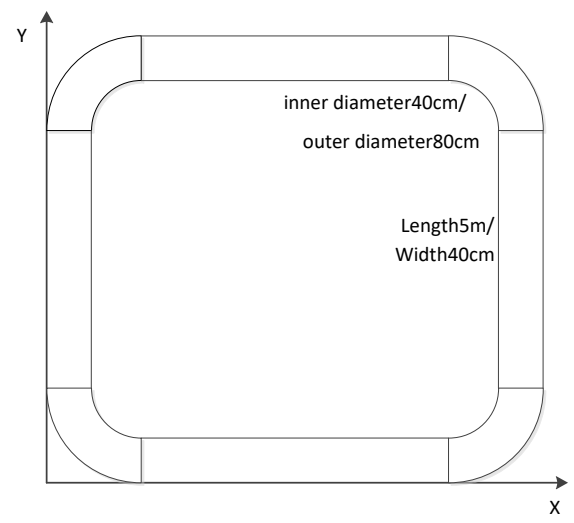

Fig. 3: Simulation racetrack.

\subsection{Simulation experiment}

The simulation model whose wheelbase length of about $21 \mathrm{~cm}$, wheel spacing of about $14 \mathrm{~cm}$, the car weighs about $2 \mathrm{~kg}$.

In this simulation experiment, the two-stage PID control parameters are adopted, with the error $7 \mathrm{~cm}$ as the cut-off point. The speed of the intelligent car in the straight and the curve is fixed. At the same time, in order to simplify the calculation, When the error information is the same, then the smart car into the corners of the time; position information calculation interval time Fixed to $30 \mathrm{~ms}$, the delay time is fixed to $10 \mathrm{~ms}$. As shown in Table I for the smart car simulation operating parameters.

TABLE I: SIMULATION PARAMETERS

\begin{tabular}{cccccccc}
\hline & PID 1 & PID 2 & Straight & Turning & Time & $\begin{array}{c}\text { Positive } \\
\text { Error }\end{array}$ & $\begin{array}{c}\text { Negative } \\
\text { Error }\end{array}$ \\
\hline 1 & $7 / 3 / 4$ & $7.8 / 4 / 5$ & $1.4 \mathrm{~m} / \mathrm{s}$ & $1.0 \mathrm{~m} / \mathrm{s}$ & $22.56 \mathrm{~s}$ & 109.87 & 112.56 \\
\hline 2 & $7.5 / 3 / 4.2$ & $8 / 3 / 5$ & $1.4 \mathrm{~m} / \mathrm{s}$ & $1.0 \mathrm{~m} / \mathrm{s}$ & $22.02 \mathrm{~s}$ & 118.94 & 117.05 \\
\hline 3 & $8.0 / 3.5 / 4.5$ & $8.8 / 3.5 / 5.5$ & $1.8 \mathrm{~m} / \mathrm{s}$ & $1.2 \mathrm{~m} / \mathrm{s}$ & $17.76 \mathrm{~s}$ & 125.53 & 125.09 \\
\hline 4 & $8.5 / 3.5 / 5$ & $9 / 3.5 / 5.5$ & $1.8 \mathrm{~m} / \mathrm{s}$ & $1.2 \mathrm{~m} / \mathrm{s}$ & $16.88 \mathrm{~s}$ & 128.33 & 130.03 \\
\hline 5 & $9.0 / 4 / 5.5$ & $9.5 / 4 / 6$ & $2.0 \mathrm{~m} / \mathrm{s}$ & $1.6 \mathrm{~m} / \mathrm{s}$ & $13.35 \mathrm{~s}$ & 134.86 & 133.22 \\
\hline 6 & $9.5 / 4 / 5.5$ & $10 / 4 / 6$ & $2.0 \mathrm{~m} / \mathrm{s}$ & $1.6 \mathrm{~m} / \mathrm{s}$ & $13.04 \mathrm{~s}$ & 139.65 & 138.98 \\
\hline
\end{tabular}

\subsection{Results comparison}

Lu Zhengchen et al. Added the S-curve and the cross-bend in his simulation experiment, but the real simulation track was not released.

In accordance with the different environments, competitive sports car speed is generally between $1.2 \mathrm{~m} /$ $\mathrm{s} \sim 2 \mathrm{~m} / \mathrm{s}$, while the simulation speed in Figure 6 only $0.4 \mathrm{~m} / \mathrm{s}$, This data has no actual reference value.

Wu Qiuxuan et al. In his paper only introduced the intelligent vehicle theory control algorithm, as the MRDS platform has integrated most of the intelligent vehicle control algorithm, so the user is only in the use of input-related motion parameters can be; at the same time because the car model is fixed, therefore, MRDS simulation platform scalability received some restrictions.

In contrast, the simulation platform proposed in this paper, because of the use of a real smart car as a research object, combined with the real data, can be considered related to the operational data has a certain practical reference value.

\section{References}

[1] You Xiaoling, Wang Nengcai. A Study on the Speed Control System of the IntelligentVehicle Based on RBF Neural Network[J]. Automatic Control Systems \& Equipments,2015,37(1):102-110 
[2] Liu Shihong, Dang Chaoliang, Wang Nengcai. Study of intelligent vehicle servo control system based on PID neural network[J]. Industrial Instrumentation \& Automation,2012,(6):97-101

[3] Jiang Shanhe, Shen Dongri. An Adaptive PID Intelligent Controller Based on Fuzzy Neural Network[J]. Journal of Anqing Teachers College (Natural Science ),2004,10(2):1-4

[4] Zheng Yi, Wang Nengcai. Improved single neuron adaptive PID control algorithm in speed control for intelligent vehicle[J]. Automation and Instrumentation,2015,(3):98-99

[5] Wang Yu, Dong Hui, Xu Jianming. A New Control Strategy for Intelligent Vehicle[J]. Journal of Zhejiang University of Technology,2012,40(2):217-221

[6] Fang Xing, Yang Ming, Peng Xinrong. Design and implementation of hardware-in-the-loop simulation system for intelligent vehicle[J]. J .H uazhong Univ .o f Sci .\& Tech.(Natr ual Science Editio n), , 2008, 36(S1):258-261.

[7] Zhou Bin, Jiang Dinan, Huang Kaisheng. Intelligent Vehicle Simulation System Based on VI-Technology[J]. Electronic Engineering \& Product World, 2006(3): 132-134.

[8] Lu Zhengchen, Yang Ming, Wang Bing. Design and implementation of simulation platform for electromagnetic guided intelligent vehicle[J]. J .H uazhong Univ .o f Sci .\& Tech.(Natr ual Science Editio n),2011, 39(Z II): 388391.

[9] Wu Qiuxuan, Zhao Junxiao, Liu Bichuan. Design and implementation of simulation platform for intelligent vehicle with camera[J], 2013, 41(Z I): 392-395.

[10] Yu Yue1, Kamel Abdelkader El1, Gong, Guanghong. Modeling intelligent vehicle agent in virtual reality traffic simulation system[C]. France: IEEE ICSCS 2013, 2013. 274-279

[11] Chungen, Hung, Abdulrahman, Yarali. Wireless Services and Intelligent Vehicle Transportation Systems[C]. Canada:IEEE CCECE 2011, 2011. 52-57

[12] J, M, Collado, C, Hilario, A, de, la, Escalera. Model Based Vehicle Detection for Intelligent Vehicles[C]. Italy:2014 IEEE Intelligent Vehicles Symposium, 2014. 572-577

[13] Chunzhao, Guo, Seiichi, Mita. A Semantic Graph of Traffic Scenes for Intelligent Vehicle Systems[J]. INTELLIGENT TRANSPORTATION SYSTEMS, 2012, (1): 57-62

[14] Wang Gongcheng. Analysis and Evaluation Platform for Front wheel Shimmy System[D]. Hefei, HeFei University of Technology),2014

[15] Guo Konghui, Liu Qing. Tire Models for Vehicle Dynamics Analysis in Steady State Condition[J]. Automotive Engineering, 1998,20(3):129-134

[16] Dong Gang. Research and Development of the Intelligent Automobile Kinetic Control System[D]. Xi'an, Xi`an University of Science and Technology

[17] Intelligent Secretary Department. Racing Rules [EB/OL]. [2016-11-1]. http:// http://www.smartcar.au.tsinghua.edu.cn.

[18] NXP. KL46 Sub-Family Reference Manual [EB/OL]. [2013-07-03]. http://www.nxp.com/cn 